\title{
Magnetic field strength and orientation effects on Co-Fe discontinuous multilayers close to percolation
}

\author{
H. G. Silva,,${ }^{1,2}$ A. M. Pereira, ${ }^{1}$ J. M. Teixeira, ${ }^{1}$ J. M. Moreira, ${ }^{1}$ G. N. Kakazei,${ }^{1,3}$ J. P. Araújo, ${ }^{1}$ Yu. G. Pogorelov,${ }^{1}$ \\ J. B. Sousa, ${ }^{1}$ M. E. Braga, ${ }^{1}$ B. Raquet,${ }^{4}$ H. Rakoto, ${ }^{4}, *$ C. Gatel, ${ }^{5}$ E. Snoeck, ${ }^{5}$ S. Cardoso,${ }^{6}$ and P. P. Freitas ${ }^{6}$ \\ ${ }^{1}$ IFIMUP and IN-Institute of Nanoscience and Nanotechnology, Departamento de Física, Universidade do Porto, \\ Rua Campo Alegre 687, 4169-007 Porto, Portugal \\ ${ }^{2}$ Geophysical Centre of Évora and Physics Department, ECT, University of Évora, Rua Romão Ramalho 59, 7002-554 Évora, Portugal \\ ${ }^{3}$ Institute of Magnetism, NAS of Ukraine, $36 \mathrm{~b}$ Vernadskogo Boulevard, 03142 Kiev, Ukraine \\ ${ }^{4}$ Laboratoire National des Champs Magnétiques Pulsés (LNCMP), 143 Av. de Rangueil, F-31432 Toulouse, France \\ ${ }^{5}$ CEMES, CNRS, 29 rue J. Marvig, F-31055 Toulouse Cedex, France \\ ${ }^{6}$ INESC-MN and IN-Institute of Nanoscience and Nanotechnology, Rua Alves Redol, 9-1, 1000-029 Lisboa, Portugal
}

(Received 1 August 2010; revised manuscript received 16 September 2010; published 21 October 2010)

\begin{abstract}
Magnetization and magnetoresistance in function of the magnitude and orientation of applied magnetic field were studied in $\mathrm{Co}-\mathrm{Fe}$ discontinuous multilayers close to their structural percolation. The high pulsed magnetic fields up to $33 \mathrm{~T}$ were used in the $120-310 \mathrm{~K}$ temperature range. Comparison between longitudinal and transverse (with respect to the film plane) field configurations was made in the low-field and high-field regimes in order to clarify the nature of the measured negative magnetoresistance. Coexistence of two distinct magnetic fractions, superparamagnetic (SPM, consisting of small spherical Co-Fe granules) and superferromagnetic (SFM, by bigger Co-Fe clusters), was established in this system. These fractions were shown to have different relevance for the system magnetization and magnetotransport. While the magnetization is almost completely (up to $\sim 97 \%$ ) defined by the SFM contribution and practically independent of temperature (in this range), the magnetoresistance experiences a crossover from a regime dominated by Langevin correlations (suppressed with temperature) between neighbor SPM and SFM moments at low fields, to that dominated by spin scattering (enhanced with temperature) of charge carriers within SFM clusters at high fields. Also, the demagnetizing effects, sensitive to the field orientation, were found to essentially define the low-field behavior and characteristic crossover field.
\end{abstract}

DOI: 10.1103/PhysRevB.82.144432

PACS number(s): 75.47.- m, 75.70.Cn, 75.75.-c

\section{INTRODUCTION}

One important class of magnetic nanostructures is nanogranular layered magnetic films, ${ }^{1-4}$ consisting of wellseparated layers of closely spaced ferromagnetic nanoparticles within a nonmagnetic matrix. Most commonly they are realized in the form of discontinuous metal-insulator magnetic multilayers (DMIMs). This type of structures displays relevant properties for spintronics applications, in particular, a relatively high tunnel magnetoresistance (TMR) for optimized composition and nanogranular structure, with special sensitivity to low (or moderate) magnetic fields (LF regime). Recently, a striking effect of resistive switching by up to four orders of magnitude was found in such systems, ${ }^{5}$ indicating their possible use in future charge memory or logics devices. Also, due to simple preparation techniques and easy manipulation of both magnetic and transport properties, by varying the nominal thickness $t$ of the magnetic layer (i.e., the thickness that a continuous layer would have to contain the same amount of material), these materials are quite promising and attract an intensive research effort for more than a decade. ${ }^{6-15}$ It was generally recognized that the main factor defining granular structure with sharp enough size distribution around certain average diameter $d$ of almost spherical granules is a proper material choice of metal/insulator pair with sufficiently high solution enthalpy to assure the nonwetting condition. In this aspect, while the classical transition metals ( $\mathrm{Co}, \mathrm{Fe}$, and their alloys) behave very similarly in these pairs, the decisive choice is for the insulating material, and the early used $\mathrm{HfO}_{2}$ (Ref. 16) or $\mathrm{SiO}_{2}$ (Ref. 2) were later succeeded by the alumina, $\mathrm{Al}_{2} \mathrm{O}_{3}$, having notably higher interface energy with magnetic metals. At least, recent advances in high performance magnetic tunnel junctions with $\mathrm{MgO}$ insulating spacers ${ }^{17}$ suggest including also this material in DMIM structures (possibly as intergranular spacers in combination with $\mathrm{Al}_{2} \mathrm{O}_{3}$ interlayer spacers).

Notably, the structural and magnetic states of $\mathrm{CoFe} / \mathrm{Al}_{2} \mathrm{O}_{3}$ DMIMs were found to display three main transitions when varying $t$ from $\sim 0.7 \mathrm{~nm}$ (a diluted granular structure with $d \approx 3 \mathrm{~nm}$ ) to $\sim 2.0 \mathrm{~nm}$ (almost continuous film) at fixed (and high enough) temperature. ${ }^{6,18,19}$ The first one, of magnetic origin, consists in passing from superparamagnetic (SPM) to the so-called superferromagnetic (SFM) state, where a net magnetization $M$ emerges (under a weak applied field) controlled by the dipolar correlations between randomly distributed close magnetic clusters. ${ }^{20}$ This process, referred to as magnetic percolation, takes place at $t \approx 1.3 \mathrm{~nm}$ for room temperature in Ref. 18 . The SFM magnetization $M$ is enhanced with growing $t$ of DMIM which finally undergoes another magnetic transition, to the common exchange ferromagnetic (FM) state at $t \approx 1.7 \mathrm{~nm}$ with almost saturated magnetization, $M \approx M_{s} .{ }^{19}$ This is immediately followed by the transition from activated to metallic electrical conduction, controlled by the structural percolation of metallic granules in the insulating matrix, near $t=1.8 \mathrm{~nm} .{ }^{6}$ Focusing on the last two transitions, one notices various as- 
pects yet to be studied in more detail, mainly related to the SFM/FM transition. In order to verify the existence or absence of a FM state below this transition point we choose to study only the case of $t=1.6 \mathrm{~nm}$ (surely below the structural percolation).

To our knowledge, all the previous studies on DMIM structures were restricted to the LF regime, $B \lesssim 5$ T. Commonly, negative MR typical of DMIM is controlled by the alignment of magnetic moments of neighbor monodomain granules by the magnetic field. Due to the spin-dependent transport effects, such alignment facilitates charge carrier tunneling and so reduces resistance vs the zero-field case (where the moments are noncorrelated); commonly this MR is believed to be a function of the net magnetization only. ${ }^{21}$ Nevertheless, in the high magnetic field (HF) regime, $B \gtrsim 5 \mathrm{~T}$, far above the magnetization saturation (see below), we found a linear (nonsaturating) field behavior of negative MR, difficult to be understood within the above approach. Apparently, the origin of MR in the HF regime should somehow differ from the above indicated correlation mechanism in the LF case. In fact, a similar linear MR behavior extended over both LF and HF ranges was already found in thin (but continuous) $\mathrm{Fe}, \mathrm{Co}$, and $\mathrm{Ni}$ films, ${ }^{22,23}$ where it was attributed to a reduction in electron-magnon scattering processes at decreasing occupation numbers of spin waves with growing field, in the framework of band ferromagnetism. ${ }^{24}$ Of course, this scattering mechanism does not seem relevant at dominating tunneling transport in the present DMIM structure. Here MR rather comes from incomplete correlations between certain "less-aligned" magnetic moments (though these correlations are almost irrelevant for the net magnetization, see below), as expected for the LF regime. However, with growing field strength, the correlation contribution to magnetoresistance, $\mathrm{MR}_{c o r}$, should attain its saturation. Therefore the contribution due to scattering mechanism, $\mathrm{MR}_{s c}$, can become prevailing in the HF MR behavior, provided a sensible part of the overall resistance is due to metallic conduction through bigger clusters (of sizes above the bulk mean-free path). Such LF-HF crossover should be characteristic just for a DMIM system close to percolation.

Another important aspect is that most of the studies on DMIM were restricted to the longitudinal magnetotransport geometry where both current and magnetic field are in the sample plane. Due to the layered DMIM structure, this geometry favors intraplane dipolar interactions and facilitates in-plane rotation of the magnetization. An alternative examination of the transverse magnetotransport geometry (with current in plane and magnetic field perpendicular to the plane) should reveal how the out-of-plane rotation of magnetization develops, highlighting the role of demagnetizing fields and weaker interplane dipolar interactions. Moreover, the above indicated LF-HF crossover turns to be sensitive to the field geometry (it moves to higher fields in the transverse geometry, see below).

From the above considerations, the main focus in this work is on two less explored issues: (i) MR response to low fields in the transverse geometry; (ii) high-field MR studies in both geometries. Also, we analyze formation of longitudinal and transverse magnetization in function of applied field and its geometry for various temperatures in the DMIM system close to percolation.



FIG. 1. (Color online) (a) A fragment of high-resolution transmission electron microscopy on the $t=1.6 \mathrm{~nm} \mathrm{CoFe} / \mathrm{Al}_{2} \mathrm{O}_{3}$ granular layer sample. (b) A contrasted sketch of the same fragment with indication of a typical tunnel conduction path (arrows) and local environments (ellipses) around SFM (left) and SPM (right) elements. (c) Schematic of longitudinal and transverse geometries of magnetoresistance measurements. (d) "Granule-in-the void" model treatment of SPM and SFM magnetic moments (white arrows) under local fields formed by the average SFM magnetization (black arrows) and by the demagnetization in the respective local cavity (its longer principal axis making the angle $\varphi_{B}$ with the global SFM order).

\section{EXPERIMENTAL}

The prepared DMIM systems consist of ten $\mathrm{Al}_{2} \mathrm{O}_{3} / \mathrm{Co}_{80} \mathrm{Fe}_{20}$ bilayers with the last capping $\mathrm{Al}_{2} \mathrm{O}_{3}$ layer. All the $\mathrm{Al}_{2} \mathrm{O}_{3}$ interlayers are of $\approx 4.0 \mathrm{~nm}$ thickness, the capping layer is $\approx 3.0 \mathrm{~nm}$ thick, and the $\mathrm{Co}_{80} \mathrm{Fe}_{20}$ nominal thickness $t$ is varied in different samples. The films are deposited on glass substrates, using Xe-ion beam sputtering. ${ }^{4}$ In this work, we focus on the samples with nominal thickness $t=1.6 \mathrm{~nm}$, also including a single-layer sample, to permit high-resolution transmission electron microscopy (HRTEM) studies. These were done on the CEMES-CRNS TECNAI F20 microscope, operating in the plane-view regime with $0.12 \mathrm{~nm}$ resolution. The high-field magnetoresistance measurements in the temperature range of 120-310 K were performed in the LNCMP facilities, Toulouse, using a $40 \mathrm{~T}$ pulsed field with a $1 \mathrm{~s}$ total pulse duration. The measurements were done in the field range up to $\sim 33 \mathrm{~T}$. MR was measured both in longitudinal (current parallel to the field, $I \| B$ ) and transverse (current perpendicular to the field, $I \perp B$ ) geometries, using the common ac techniques for lowlevel signals. The magnetization curves (at various temperatures) were taken with a Quantum Design MPMS-5 superconducting quantum interference device (SQUID), at IFIMUP, Porto.

\section{RESULTS}

A typical sample of HRTEM images is shown in Fig. 1(a). The bright patches correspond to $\mathrm{Al}_{2} \mathrm{O}_{3}$ and the dark patches to $\mathrm{Co}_{80} \mathrm{Fe}_{20}$ regions, revealing formation of wormlike $\mathrm{Co}_{80} \mathrm{Fe}_{20}$ islands embedded into the $\mathrm{Al}_{2} \mathrm{O}_{3}$ matrix with aver- 
age size of $\approx 30 \mathrm{~nm}$ but having a rather broad distribution of sizes (from $\sim 3$ up to $\sim 100 \mathrm{~nm}$ ) and shapes (from almost spherical up to multiply ramified). This topology characterizes closeness of our DMIM to structural percolation. However, the presence of a considerable number of tunnel bridges between the $\mathrm{Co}_{80} \mathrm{Fe}_{20}$ clusters and of a residual fraction of small isolated $\mathrm{Co}_{80} \mathrm{Fe}_{20}$ granules [see Fig. 1(b)], like the dominating fraction in diluted DMIM with $t \leqslant 1.0 \mathrm{~nm}$, gives rise to diverse transport and magnetic properties discussed formerly. ${ }^{6}$ In particular, it was shown that the temperature dependence of the electrical resistance for DMIM structures with $t=1.6 \mathrm{~nm}$ roughly follows the Sheng and Abeles law, ${ }^{25}$ pointing the thermally activated charge carrier tunneling as the dominant resistive process. The same behavior with typical resistance values of $\sim 50 \mathrm{k} \Omega$ and $\sim \exp \left(2 \sqrt{C / k_{\mathrm{B}} T}\right)$ temperature law were found in the present study. A rather low Sheng-Abeles activation constant, $C \approx 0.1 \mathrm{meV}$, for tunneling at average distance $s \sim 2 \mathrm{~nm}$ with inverse decay length $\chi \sim 10 \mathrm{~nm}^{-1}$, corresponds to a very low activation energy $E_{a} \approx C /(\chi s) \sim 5 \mu \mathrm{eV}$ which, being otherwise estimated as $E_{a} \sim e^{2} /\left(\varepsilon_{\text {eff }} a\right)$ for a charged particle of $a \sim 30 \mathrm{~nm}$ size, indicates a strong screening effect on it from neighbor particles, making the effective dielectric constant as high as $\varepsilon_{\text {eff }} \sim 10^{4}$ for this composition. These and other properties can be qualitatively and semiquantitatively understood, modeling the system as a mixture of two interpenetrated nanostructures: (i) small and almost spherical granules of $d \approx 4 \mathrm{~nm}$ average diameter that behave as SPM (less correlated) moments, each subject to a local internal field $\mathbf{B}_{i}=\mathbf{B}+\mathbf{B}_{d}$ with $\mathbf{B}_{d}$ due to demagnetizing effects of its closest environment and (ii) a network of bigger wormlike clusters of $a \sim 30 \mathrm{~nm}$ average length whose moments are almost aligned to form the SFM magnetization. The relative numbers of SPM and SFM moments can be visually estimated from Fig. 1(b) as $n_{\mathrm{SPM}}: n_{\mathrm{SFM}}=1: 3$ (by the direct counting of 19 SFM clusters vs 6 SPM elements), so about $97 \%$ of magnetic material pertains to the SFM subsystem which thus dominates in the overall magnetization.

On the other hand, the relative numbers $n_{\mathrm{SFM}-\mathrm{SFM}}$, $n_{\text {SFM-SPM }}$, and $n_{\text {SPM-SPM }}$ of tunnel bridges between pairs of neighbor SFM-SFM, SFM-SPM, and SPM-SPM elements along most favorable conducting paths are again visually estimated from Fig. 1(b) as 16:10:1, so the magnetotransport properties are mainly defined by the first two types of bridges. Since the tunneling probability in a pair, $P_{i j} \propto E_{i j} /\left[\exp \left(E_{i j} / k_{\mathrm{B}} T\right)-1\right]$, turns to be almost insensitive to low activation energies $E_{i j} \ll k_{\mathrm{B}} T(i, j=\mathrm{SFM}, \mathrm{SPM})$ in the studied temperature range, the overall resistance and magnetoresistance should be mainly defined by the SFM-SFM and SFM-SPM bridges.

Below, the MR data for the two geometries are presented in Fig. 2 and analyzed separately for the LF and HF regimes (being plotted with reduced sampling rate compared to the very high original rate of the pulsed field measurements, for the viewer's convenience). Then it is followed by the SQUID magnetization data in magnetic fields up to $\sim 5 \mathrm{~T}$.

\section{A. Low-field magnetoresistance}

Comparison between the LF MR in longitudinal and transverse geometries [insets in Figs. 2(a) and 2(b)] reveals a
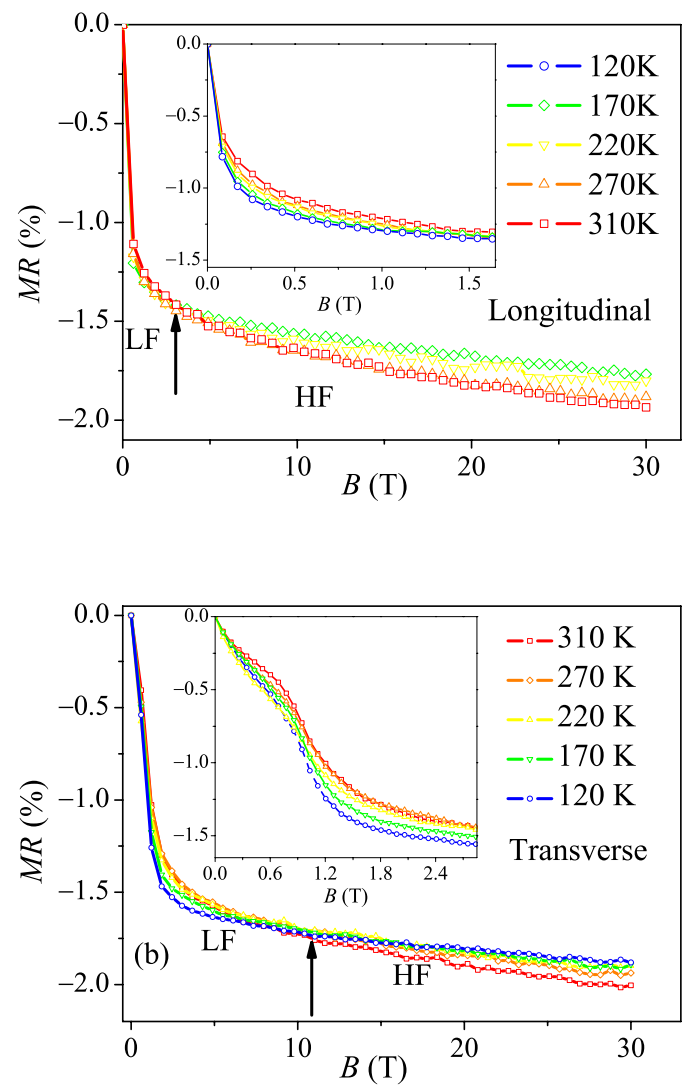

FIG. 2. (Color online) DMIM magnetoresistance in longitudinal and transverse geometry.

notable difference between the two for all the temperatures considered. This is yet more clearly seen in the corresponding plots of the MR field derivative, $\partial \mathrm{MR} / \partial B$ in function of $B$, presented in Fig. 3. In the longitudinal case, they decay monotonously from a high initial value to a low asymptotic HF value [Fig. 3(a)], while in the transverse case they display a much lower initial value and a notable zigzag anomaly around $B \sim 0.4 \mathrm{~T}$ [Fig. 3(b)] (a weak apparent zigzag anomaly at $\sim 0.2 \mathrm{~T}$ in the longitudinal case is most probably instrumental due to a small mismatch at the change of measurement scales). Also the temperature effect on $\partial \mathrm{MR} / \partial B$ (changing from negative to positive with growing field) is much more pronounced in the transverse case. The physical processes and characteristic field scales for these LF anomalies, together with similarity of asymptotic HF values for both geometries will be discussed below in the framework of a specific model for DMIM magnetic state. It is worth to mention that the continuous field MR measurements in LF regime (not shown) agree with the presented pulsed field data.

\section{B. High-field magnetoresistance}

The MR data in HF regime are displayed in the main panels of Fig. 2. As discussed above, the negative magnetoresistance for this regime is expected from important effects of spin-diffusion scattering within band ferromagnetism like those reported by Raquet et al. ${ }^{22,23}$ They were able to 

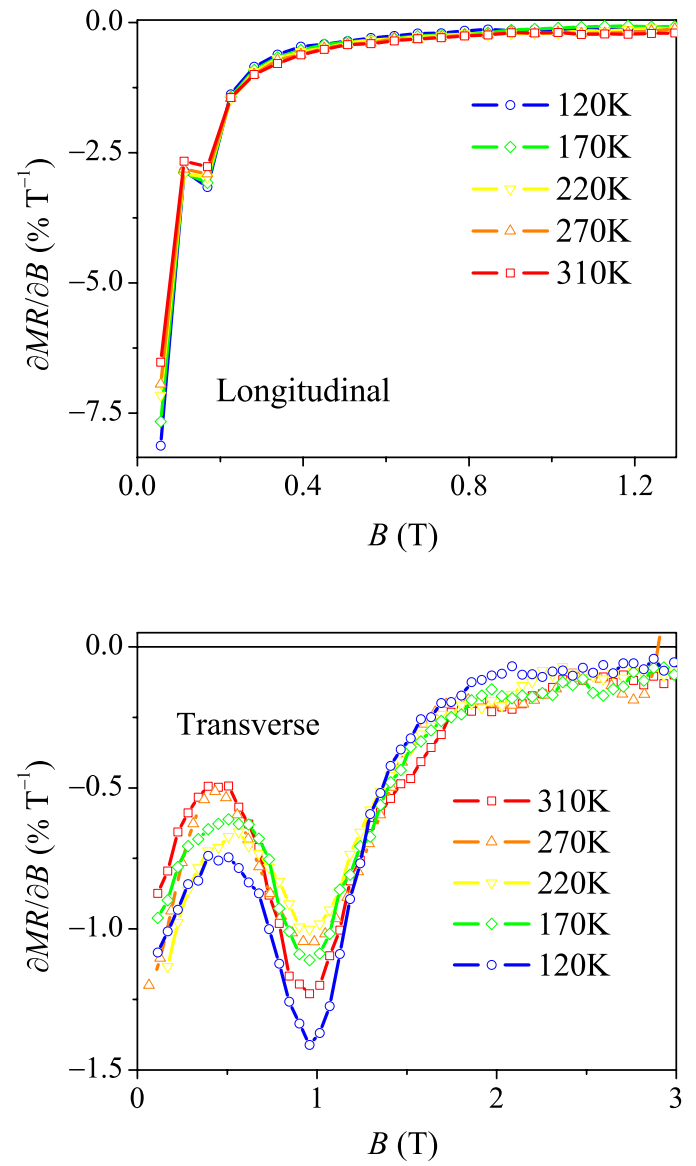

FIG. 3. (Color online) Field derivative of magnetoresistance in longitudinal and transverse geometry.

estimate the magnetic contribution (field dependent) to resistivity, $\rho_{\text {mag }}(T, B)$, in $3 d$ ferromagnets from electron-magnon scattering [which produces both magnon mass renormalization and HF MR (Ref. 23)] improving significantly the previous Goodings' work. ${ }^{24}$ The main spin-flip processes considered by those authors were intraband $d$ - $d$ and interband $s$ - $d$ transitions while the intraband $s-s$ ones have a negligible contribution to the resistivity. Within such assumptions, a quasilinear HF MR dependence of $\propto B T \ln \left(\mu_{\mathrm{B}} B / k_{\mathrm{B}} T\right)$ type, giving an almost linear temperature dependence of the HF MR derivative $\partial \mathrm{MR} /\left.\partial B\right|_{B \geq \mu_{0} M_{s}}$ for high enough temperatures, were found. These dependencies are also verified in the present HF measurements for both geometries, Figs. 2(a) and 2(b), indicating that band ferromagnetism phenomena are responsible for the nonsaturating HF MR in our DMIM. In addition, the HF behavior of MR turns to be very similar for the two geometries, with typical values $\partial \mathrm{MR} / \partial B \sim 10^{-4} \mathrm{~T}^{-1}$, near room temperature. This is a clear indication that these processes are not affected significantly neither by the specific topology of the films nor by the magnetic field direction. In fact, this is one of the most important results of the present work that clearly attests for a "band" explanation of the HF MR and obviously supports the presence of FM behavior already for $t=1.6 \mathrm{~nm}$. Here bandlike electron-magnon interactions most probably occur inside the bigger wormlike clusters where the electronic mean-free path is large enough to enable them feeling the short-range ex-
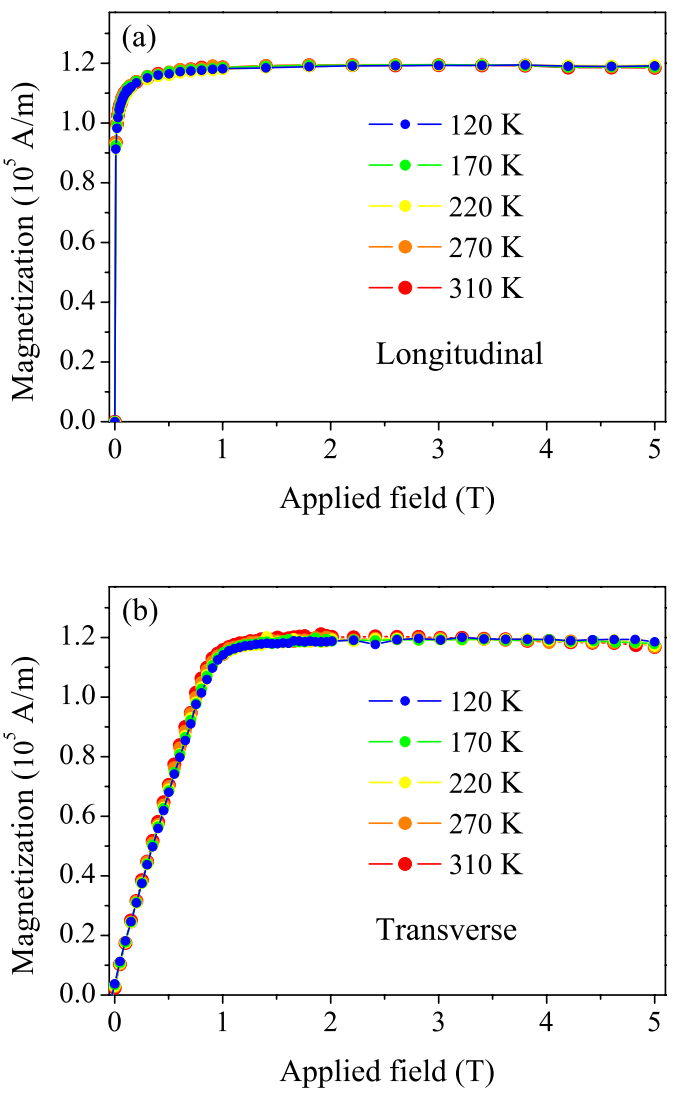

FIG. 4. (Color online) DMIM magnetization in longitudinal and transverse geometry. Note the collapse of the data for all temperatures on the same curve.

change interactions. Then the related contribution becomes dominating in the field dependence of MR at high enough fields where the above considered TMR contribution gets almost saturated.

However, our HF MR data also present some new features, compared to the case of continuous film in Refs. 22 and 23. Namely, the derivative $\partial \mathrm{MR} /\left.\partial B\right|_{B \geq \mu_{0} M_{s}}$ as a function of temperature exhibits a tendency to pronounced nonzero limit at $T \rightarrow 0$, suggesting yet additional, nonmagnonic scattering MR mechanism to exist in DMIM systems. This mechanism will be further discussed below. Meanwhile, in a previous study on DMIM of this nominal thickness $t$ (Ref. 19) no anisotropic MR was detected, which was interpreted as irrelevance of the Lorentz contribution to resistivity. In the framework of the LF-HF crossover, this can be understood as insignificance of the corresponding $\sim B^{2}$ contribution to resistivity both in the LF regime (besides the TMR contribution) and in the $\mathrm{HF}$ regime (besides the scattering contribution).

\section{Magnetization}

The magnetization curves in function of the applied field $M(B)$ for both configurations at different temperatures are presented in Figs. 4(a) and 4(b). Their high-field saturation was assured after subtraction of a significant diamagnetic contribution from the glass substrate and a smaller paramag- 

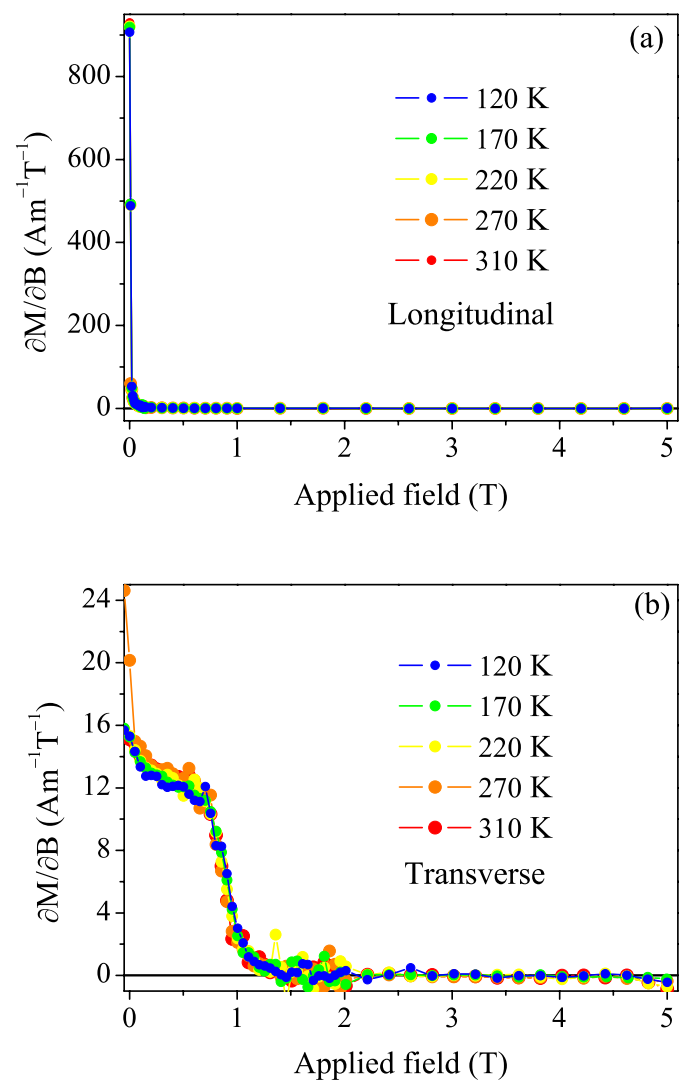

FIG. 5. (Color online) Field derivative of DMIM magnetization in longitudinal and transverse geometry.

netic contribution from the amorphous $\mathrm{Al}_{2} \mathrm{O}_{3}$ matrix. In fact, the LF $M(B)$ results display a good correlation with the above LF MR data. Thus, the longitudinal $M$ curves show (besides an abrupt initial jump by the SFM contribution) a fast saturation in low fields as is also clearly seen in the MR data. Furthermore, the behavior of the transverse magnetization is also in a good agreement with transverse LF MR. In particular, the LF magnetization derivative, $\partial M /\left.\partial B\right|_{B \lesssim \mu_{0} M_{s}}$, presented in Fig. 5 displays a shoulder anomaly within the same field region as the zigzag anomaly in magnetoresistance derivative, $\partial \mathrm{MR} /\left.\partial B\right|_{B \lesssim \mu_{0} M_{s}}$ in Fig. 3. Now, in order to explain qualitatively the observed MR and $M$ behaviors in concordance with the DMIM structure parameters, a simple model for LF and HF magnetic states is proposed and analyzed.

\section{MODEL AND DISCUSSION}

The understanding of the observed magnetic behaviors in longitudinal and transverse cases can be obtained from interplay between the effects of local shape anisotropy for SPM and SFM nanoelements and of demagnetization in the discontinuous magnetic layer. An important specifics of DMIM system close to structural percolation consists in a possibility to consider the nonmagnetic spacer around each magnetic element as a "void" in the SFM background of magnetization $\mathbf{M}_{\mathrm{SFM}}$ (per unit total volume of a layer) or otherwise as a negative magnetization $\mathbf{M}_{\mathrm{SFM}}$ in the volume of a void super- imposed over the uniform SFM background (in the spirit of Lorentz cavity). For an SPM element, this "granule-in-void" model defines the overall demagnetizing field $\mathbf{B}_{d}$ on it as a sum of contributions from the positively magnetized background and from the negatively magnetized void: $\mathbf{B}_{d}=\mathbf{B}_{b}+\mathbf{B}_{v}$. Here $\mathbf{B}_{b}=-\hat{N}_{b} \mathbf{M}_{\mathrm{SFM}}$ includes the evident demagnetizing tensor $\hat{N}_{b}=(0,0,1)$, only nonzero in the normal direction to plane, while $\mathbf{B}_{v}=\hat{N}_{v}=\mathbf{M}_{\mathrm{SFM}}$ includes the "magnetizing" tensor $\hat{N}_{v}=\left(n_{1}, n_{2}, 1-n_{1}-n_{2}\right)$, with the factors $n_{1,2}$ being specific to a particular void shape (assumed elliptical) in the local frame referred to its particular in-plane orientation $\varphi_{B}$ [see Fig. 1(c)]. Thus, the related "magnetizing" field $\mathbf{B}_{v}$ effectively accounts for the magnetostatic coupling of SPM element with its environment. The mean-field treatment of such system defines the orientation of the SFM magnetization $\mathbf{M}_{\mathrm{SFM}}=M_{\mathrm{SFM}}(\sin \theta \cos \varphi, \sin \theta \sin \varphi, \cos \theta)$ (in the laboratory frame) from the minimum of the corresponding energy: $E_{\mathrm{SFM}}=\frac{1}{2} \mu_{0} \mathbf{M}_{\mathrm{SFM}} \hat{N}_{b} \mathbf{M}_{\mathrm{SFM}}-\mathbf{B} \cdot \mathbf{M}_{\mathrm{SFM}}$. Here the same average values of $n_{1,2}$ are assumed for each SPM element to obtain its thermal-averaged magnetic moment as $\langle\mathbf{m}\rangle=m L\left(m B_{i} / k_{\mathrm{B}} T\right) \mathbf{B}_{\mathbf{i}} / B_{i}$, where $m=M_{s} \pi d^{3} / 6$, the Langevin function $L(x)=\operatorname{coth} x-1 / x$, and the local field is a function of the void orientation angle: $\mathbf{B}_{i}\left(\varphi_{B}\right)$. Finally, after averaging in random orientations of voids around SPM elements (within the positive half circle with respect to the in-plane $\mathbf{B}$ projection), the thermal+structural averaged magnetic moment per granule results as

$$
\langle\overline{\mathbf{m}}\rangle=m \int_{-\pi / 2}^{\pi / 2} d \varphi L\left[m B_{i}(\varphi) / k_{\mathrm{B}} T\right] \mathbf{B}_{i}(\varphi) / B_{i}(\varphi) .
$$

Evidently, a similar mean-field treatment can be also developed for the SFM elements, to describe formation and stability of the proper SFM order in the DMIM structure. This will be the subject of a separate study and now we simply suppose it to be assured within the actual temperature range (well below the related SFM Curie temperature ${ }^{4,6}$ ), defining $\mathbf{M}_{\mathrm{SFM}}$ as a certain function of $\mathbf{B}$ but almost independent of $T$. Then the magnetization $\mathbf{M}$ (per unit total volume of a layer) is written as

$$
\mathbf{M}=\mathbf{M}_{\mathrm{SFM}}+n_{\mathrm{SPM}}\langle\overline{\mathbf{m}}\rangle
$$

and the correlation part of magnetoresistance

$$
\mathrm{MR}_{c o r}(\mathbf{B})=\frac{C(\mathbf{B})-C(0)}{P^{-2}+C(\mathbf{B})}
$$

corresponds to the known Inoue-Maekawa formula, ${ }^{21}$ involving the correlators $C(\mathbf{B})=\langle\overline{\mathbf{m}}\rangle \cdot \mathbf{M}_{\mathrm{SFM}} /\left(m M_{\mathrm{SFM}}\right)$, $C(0)=\lim _{B \rightarrow 0} C(\mathbf{B})$, and the spin polarization $P$ of the magnetic metal (commonly taken about $\sim 0.4$ for bulk $\mathrm{Co}-\mathrm{Fe}$ alloy $\left.{ }^{26-28}\right)$.

Let us consider how the key term for Eqs. (2) and (3), the average SPM moment $\langle\overline{\mathbf{m}}\rangle$, is formed in the particular cases of longitudinal and transverse geometries. For the in-plane field, we have $\theta=\pi / 2, \varphi=0$, then the averaged SPM moment is calculated from Eq. (1) using the local field vector 


$$
\mathbf{B}_{i}(\varphi)=\left(\begin{array}{c}
B+\mu_{0} M_{\mathrm{SFM}}(\bar{n}-\delta \cos 2 \varphi) \\
-\mu_{0} M_{\mathrm{SFM}} \delta \sin 2 \varphi \\
0
\end{array}\right)
$$

with $\bar{n}=\left(n_{1}+n_{2}\right) / 2$ and $\delta=\left(n_{2}-n_{1}\right) / 2$.

For the normal-to-plane field, we admit its small deviation from exact normal, $\theta \ll 1$ (almost inevitable in practice and allowing to fix the in-plane field orientation, $\varphi=0)$. Then the local field is expressed as

$$
\mathbf{B}_{i}(\varphi) \approx\left(\begin{array}{c}
B \sin \theta+\mu_{0} M_{\mathrm{SFM}} \cos \theta_{B}(\bar{n}-\delta \cos 2 \varphi) \\
-\mu_{0} M_{\mathrm{SFM}} \cos \theta_{B} \delta \sin 2 \varphi \\
B(\cos \theta-2 \bar{n})
\end{array}\right)
$$

for weak enough applied fields, as far as $\theta_{B}$ $=\arctan \left(B / \mu_{0} M_{\mathrm{SFM}}\right)$ is small. For strong enough applied fields, when $\theta_{B}$ gets close to $\pi / 2-\theta$ (that is SFM moments get close to alignment with $\mathbf{B}$ ), one has

$$
\mathbf{B}_{i}(\varphi) \approx\left\{\begin{array}{c}
{\left[B+\mu_{0} M_{\mathrm{SFM}}(\bar{n}-\delta \cos 2 \varphi)\right] \sin \theta} \\
-\mu_{0} M_{\mathrm{SFM}} \delta \sin 2 \varphi \sin \theta \\
\left(B-2 \mu_{0} M_{\mathrm{SFM}} \bar{n}\right) \cos \theta
\end{array}\right\} .
$$

Thus the system of Eqs. (1)-(6) realizes a model description of the DMIM magnetization and correlation-limited magnetoresistance.

For numerical calculations using Eqs. (1)-(6) we set the parameter of saturated SFM magnetization: $\mu_{0} M_{\mathrm{SFM}} \approx 1 \mathrm{~T}$ (as for Co-Fe saturated magnetization $M_{s} \approx 1.6 \times 10^{6} \mathrm{~A} / \mathrm{m}$ and the volumetric factor $f \approx 0.5)$. For the SPM granules, we use the parameters: $n_{\mathrm{SPM}} \approx 6.4 \times 10^{23} \mathrm{~m}^{-3}$ [as for six granules in the $52 \times 45 \times 4 \mathrm{~nm}^{3}$ volume, seen in Figs. 1(a) and $1(\mathrm{~b})]$ and $m \approx 4 \times 10^{-20} \mathrm{~A} / \mathrm{m}^{2}$. This shows the maximum SPM contribution to the overall $M$ [by Eq. (2)] to be below $\sim 3 \%$ and thus safely negligible.

Considering SPM-SFM correlations, we assume for simplicity in-plane symmetry of a void around an SPM element: $n_{1} \approx n_{2} \approx 0.18$ [as by the Osborn formulas ${ }^{29}$ for an oblate spheroid of $\approx 12 \mathrm{~nm}$ diameter and $\approx 4 \mathrm{~nm}$ height, see Fig. $1(\mathrm{~b})]$. Since this assumption makes $\langle\mathbf{m}\rangle$ independent of $\varphi$, we can get rid of integration in Eq. (1): $\langle\overline{\mathbf{m}}\rangle=\langle\mathbf{m}\rangle$, and thus facilitate the calculations. Also, the best fit to LF MR data is obtained with the polarization parameter $P \approx 0.13$, sensibly lower than the bulk value, which may be due to a pronounced size effect in nanogranules.

The obtained results for longitudinal and transverse MR shown in Fig. 6 are in a fair agreement with the experimental observations. In particular, the LF anomaly in the transverse MR is qualitatively reproduced, as a result of faster initial out-of-plane deviation of the isotropic SPM moments and subsequent loss of their correlation with the slower deviating (by the strong SFM demagnetization effect) SFM moments. The characteristic field of this anomaly corresponds to the maximum SPM-SFM miscorrelation at $B \sim \mu_{0} M_{\mathrm{SFM}}$, the crossover value between Eqs. (5) and (6), in agreement with the observed behavior. Finally, the HF MR estimation in the context of electron-magnon scattering theory can qualita-
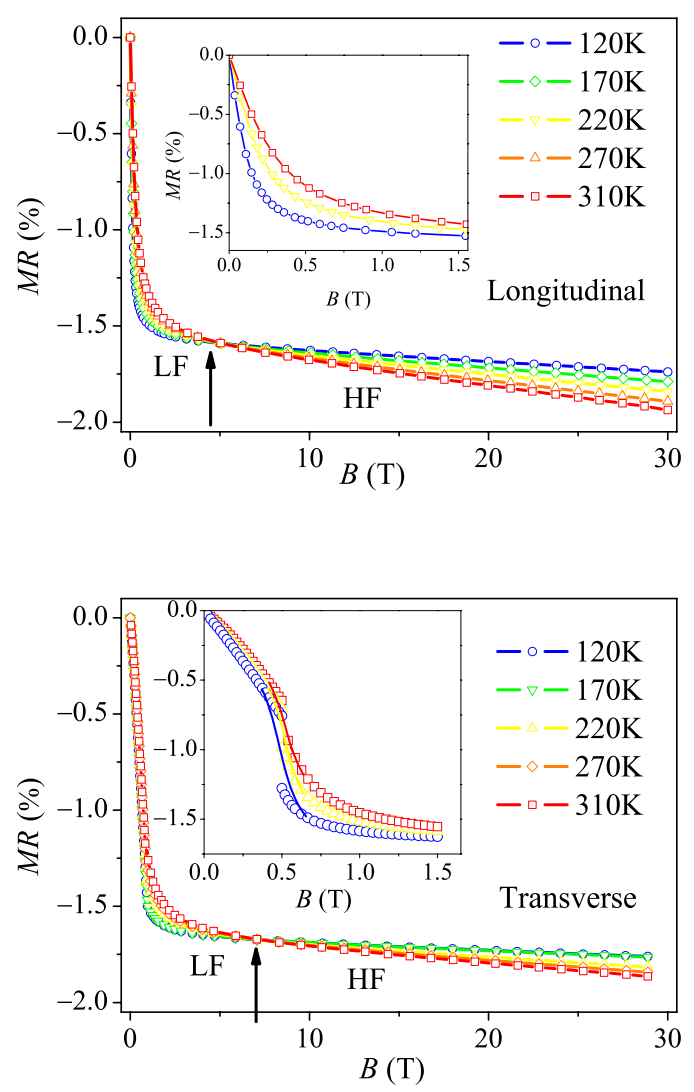

FIG. 6. (Color online) Calculated magnetoresistance of DMIM by the granule-in-void model, Eqs. (1)-(6), in longitudinal and transverse geometries. Insets show in more detail the low-field MR, including its anomaly in the transverse case as calculated from Eqs. (5) and (6) (two sets of lines with symbols) and interpolated by the guide for eyes (solid lines).

tively describe the LF-HF crossover, in particular, the passage from negative temperature effect on LF MR to the positive effect on HF MR.

However the experimental field derivative $\partial \mathrm{MR} /\left.\partial B\right|_{B \geq \mu_{0} M_{s}}$, shown in Fig. 5, tends to pronounced nonzero limit values at $T \rightarrow 0$ either in longitudinal and transverse geometries. Hence it cannot be only fitted by the electron-magnon scattering $\propto T \ln \left(k_{\mathrm{B}} T / \mu_{\mathrm{B}} B\right)$ dependence. This suggests the presence of an additional contribution to negative MR in DMIM close to percolation, compared to continuous magnetic films, which persists even at full saturation of magnetic moments, either on macroscopic scale (between different nanoelements) and on microscopic scale (in spin-polarized electronic band states). From the known magnetoresistive mechanisms in condensed matter, the only one satisfying the above criteria is that due to suppression of weak localization in disordered electronic systems by strong enough magnetic field. ${ }^{30}$ In the considered situation, weak localization can arise in closed electronic paths within a low-dimensional SFM cluster under multiple reflections from its boundaries (more effective than reflections on impurities, commonly considered in bulk systems). For typical weak localization effect on resistance: $R_{l o c} / R \sim \hbar /\left(4_{\pi \varepsilon_{\mathrm{F}} \tau}\right) \ln \left(1+\tau_{i} / \tau\right)$, with $\tau \ll \tau_{i}$ and Fermi energy $\varepsilon_{\mathrm{F}}$, we have in this case the elastic collision time 
$\tau$ as short as $\sim \ell / v_{\mathrm{F}} \sim 6 \times 10^{-14} \mathrm{~s}$ [for Fermi velocity $v_{\mathrm{F}} \approx 3.3 \times 10^{5} \mathrm{~m} / \mathrm{s}$ (Ref. 31)], so that $R_{l o c} / R$ can amount up to $\sim 0.5 \%$, a sizeable fraction of the maximum MR in our system. The magnetic field $B$ will suppress this effect if its flux through the SFM cluster, $\Phi \sim B l d$, is on the order of flux quantum $\Phi_{0}$. Then the corresponding value $B \sim \Phi_{0} /(l d) \sim 30 \mathrm{~T}$ is just a reasonable field scale for this experiment. Yet we notice that a bigger zero-temperature limit for $\mathrm{HF} \partial \mathrm{MR} / \partial B$ in longitudinal geometry can be ascribed to a bigger cross section of a cluster in this case. Otherwise, the temperature dependence of HF MR is almost linear and insensitive to geometry in concordance with the common magnonic scattering theory. Finally, the characteristic LF-HF crossover fields are estimated from the interplay of the corresponding contributions in $\mathrm{MR}=\mathrm{MR}_{c o r}+\mathrm{MR}_{s c}$, using the phenomenological form $\mathrm{MR}_{s c}=-\alpha-\beta T$ with the parameters $\alpha_{\|} \approx 1.8 \times 10^{-5} \mathrm{~T}^{-1}, \alpha_{\perp} \approx 1.5 \times 10^{-5} \mathrm{~T}^{-1}$, $\beta_{\|} \approx 3 \times 10^{-7} \mathrm{~T}^{-4} \mathrm{~K}^{-1}$, and $\beta_{\perp} \approx 2 \times 10^{-7} \mathrm{~T}^{-1} \mathrm{~K}^{-1}$, fitting the asymptotic $\mathrm{HF} \partial \mathrm{MR} / \partial B$. The resulting values of $B_{\|} \sim 3 \mathrm{~T}$ and $B_{\perp} \sim 7 \mathrm{~T}$ are also in agreement with the measured ones.

\section{CONCLUSIONS}

The magnetic and magnetotransport properties were studied in discontinuous metal-insulator $\mathrm{CoFe} / \mathrm{Al}_{2} \mathrm{O}_{3}$ multilayers. The high-resolution microscopy studies show the discontinuous $\mathrm{Co}-\mathrm{Fe}$ layers with nominal thickness $1.6 \mathrm{~nm}$ to consist of two main fractions: (i) the dominant fraction of long warmlike (including ramified) clusters of Co-Fe granules and (ii) the residual fraction of isolated almost spherical granules. The first fraction forms the so-called superferromagnetic order of magnetic moments in clusters (by their magnetostatic interactions) and thus can be called the SFM fraction; it dominates in the total magnetization, resulting almost temperature independent up to room temperature. The second fraction gives a superparamagnetic response to the internal field on a granule (including the magnetostatic field by SFM fraction) and thus can be called the SPM fraction. This fraction turns out to be decisive for the system magnetoresistance at low enough applied fields (until saturation of SPM moments), mainly defined by the SPM-SFM magnetic correlations. At higher fields, the main role in MR passes to electronic spin-scattering processes (including those due to quantum weak localization) within SFM clusters. A simple model of SPM-SFM correlations, treating a SPM moment under effective field in a local void within the SFM network, permits a reasonable description of the low-field MR behavior in this composite system. In particular, it explains the difference in the MR field and temperature dependencies for the cases when the applied field lies in the film plane and perpendicular to this plane, by the interplay of demagnetizing factors of the whole film, the local void, and the particle within void. The distinction between the observed low- and high-field MR behaviors characterizes the coexistence of superferromagnetic (between magnetic clusters) and common exchange-ferromagnetic (within clusters) order in the condensed DMIM systems. These results can be important for the design of high-density magnetic arrays in modern spintronics applications.

\section{ACKNOWLEDGMENTS}

This work was partially supported by the Portuguese FCT through Grants No. SFRH/BD/24190/2005 (H.S.), No. SFRH/BD/22373/2005 (A.P.), and No. SFRH/BD/ 24012/2005 (J.T.) and the European research program EuroMagNET under the EU under Contract No. RII3-CT2004-506239. G.N.K. acknowledges support from Portuguese FCT through "Ciência 2007" program. Authors are grateful to J. M. Santos and J. O. Ventura for fruitful discussions and support.

\footnotetext{
*Deceased.

${ }^{1}$ Ch. Morawe and H. Zabel, J. Appl. Phys. 77, 1969 (1995).

${ }^{2}$ B. Dieny, S. Sankar, M. R. McCartney, D. J. Smith, P. BayleGuillemaud, and A. E. Berkowitz, J. Magn. Magn. Mater. 185, 283 (1998)

${ }^{3}$ C. A. F. Fettar, J.-L. Maurice, F. Petroff, L. F. Schelp, A. Vaures, and A. Fert, Thin Solid Films 319, 120 (1998).

${ }^{4}$ G. N. Kakazei, P. P. Freitas, S. Cardoso, A. M. L. Lopes, Yu. G. Pogorelov, J. A. M. Santos, and J. B. Sonsa, IEEE Trans. Magn. 35, 2895 (1999).

${ }^{5}$ H. Silva H. L. Gomes, Yu. G. Pogorelov, P. Stallinga, D. M. de Leenw, J. P. Aranjo, J. B. Sonsa, S. C. J. Meskers, G. Kakazei, S. Cardoso, and P. P. Freitas, Appl. Phys. Lett. 94, 202107 (2009).

${ }^{6}$ G. N. Kakazei, Yu. G. Pogorelov, A. M. L. Lopes, J. B. Sonsa, P. P. Freitas, S. Cardoso, M. M. Pereira Azevedo, and E. Snoeck, J. Appl. Phys. 90, 4044 (2001).

${ }^{7}$ W. Kleemann, O. Petracic, Ch. Binek, G. N. Kakazei, Yu. G. Pogorelov, J. B. Sousa, S. Cardoso, and P. P. Freitas, Phys. Rev. B 63, 134423 (2001).
}

${ }^{8}$ M. Anas, C. Bellouard, and M. Vergnat, J. Appl. Phys. 96, 1159 (2004).

${ }^{9}$ C. J. Yang, M. Zhang, and Z. D. Zhang, J. Korean Phys. Soc. 43, 762 (2003)

${ }^{10}$ H. S. Hsu, J. C. A. Huang, L. Horng, C. H. Lee, Y. H. Huang, Y. F. Liao, and M. Z. Lin, IEEE Trans. Magn. 41, 903 (2005).

${ }^{11}$ R. Bručas, M. Hanson, R. Gunnarsson, E. Wahlström, M. van Kampen, B. Hjörvarsson, H. Lidbaum, and K. Leifer, J. Appl. Phys. 101, 073907 (2007).

${ }^{12}$ A. García-García, A. Vovk, J. A. Pardo, P. Štrichovanec, C. Magén, E. Snoeck, P. A. Algarabel, J. M. De Teresa, L. Morellón, and M. R. Ibarra, J. Appl. Phys. 105, 063909 (2009).

${ }^{13}$ S. W. Bie, J. J. Jiang, Q. Ma, G. Du, L. Yuan, Z. K. Feng, and H. H. He, J. Nanosci. Nanotechnol. 9, 1535 (2009).

${ }^{14}$ A. García-García, A. Vovk, J. A. Pardo, P. Štrichovanec, P. A. Algarabel, C. Magén, J. M. De Teresa, L. Morellón, and M. R. Ibarra, J. Appl. Phys. 107, 033704 (2010).

${ }^{15}$ R. Bručas, M. Hanson, P. Apell, P. Nordblad, R. Gunnarsson, and B. Hjörvarsson, Phys. Rev. B 81, 224437 (2010).

${ }^{16}$ S. Sankar, B. Dieny, and A. E. Berkowitz, J. Appl. Phys. 81, 
5512 (1997).

${ }^{17}$ S. Yuasa, A. Fukushima, H. Kubota, Y. Suzuki, and K. Ando, Appl. Phys. Lett. 89, 042505 (2006).

${ }^{18}$ G. N. Kakazei, Yu. G. Pogorelov, J. A. M. Santos, J. B. Sousa, P. P. Freitas, S. Cardoso, N. A. Lesnik, and P. E. Wigen, J. Magn. Magn. Mater. 266, 57 (2003).

${ }^{19}$ J. B. Sousa, J. A. M. Santos, R. F. A. Silva, J. M. Teix-eira, J. Ventura, J. P. Araiijo, P. P. Freitas, S. Cardoso, Yu. G. Pogorelov, G. N. Kakazei, and E. Snoeck, J. Appl. Phys. 96, 3861 (2004).

${ }^{20}$ Y. G. Pogorelov, G. N. Kakazei, M. D. Costa, and J. B. Sousa, J. Appl. Phys. 103, $07 \mathrm{~B} 723$ (2008).

${ }^{21}$ J. Inoue and S. Maekawa, Phys. Rev. B 53, R11927 (1996).

${ }^{22}$ B. Raquet, M. Viret, E. Sondergard, O. Cespedes, and R. Mamy, Phys. Rev. B 66, 024433 (2002).

${ }^{23}$ B. Raquet, M. Viret, P. Warin, E. Sondergard, and R. Mamy, Physica B 294-295, 102 (2001).
${ }^{24}$ D. A. Goodings, Phys. Rev. 132, 542 (1963).

${ }^{25}$ P. Sheng, B. Abeles, and Y. Aire, Phys. Rev. Lett. 31, 44 (1973).

${ }^{26}$ L. Gao, X. Jiang, S.-H. Yang, P. M. Rice, T. Topuria, and S. S. P. Parkin, Phys. Rev. Lett. 102, 247205 (2009).

${ }^{27}$ S. Blundell, Magnetism in Condensed Matter (Oxford University Press, New York, 2001).

${ }^{28}$ G. N. Kakazei, Yu. G. Pogorelov, J. B. Sousa, V. O. Golub, N. A. Lesnik, S. Cardoso, and P. P. Freitas, J. Magn. Magn. Mater. 226-230, 1828 (2001).

${ }^{29}$ J. P. Osborn, Phys. Rev. 67, 351 (1945).

${ }^{30}$ B. L. Altshuler, D. E. Khmernitzkii, A. I. Larkin, and P. A. Lee, Phys. Rev. B 22, 5142 (1980).

${ }^{31}$ D. Y. Petrovykh, K. N. Altmann, H. Hochst, M. Laub-scher, S. Maat, G. J. Mankey, and F. J. Himpsel, Appl. Phys. Lett. 73, 3459 (1998). 